\title{
Investigação de alterações eletrocardiográficas em gatas (Felis catus) submetidas a pneumoperitônio
}

\author{
Investigation of eletrocardiographic changes in cats (Felis catus) submitted to pneumoperitoneum \\ Jéssica Dias Ribas Tavares da Silva ${ }^{\dagger}$, José Edgard de Oliveira Alves ${ }^{\ddagger}$ Camilla Xavier Martins ${ }^{\S}$, \\ Guilherme de Souza Vieiral, Fernanda Antunes ${ }^{\circ}$
}

\begin{abstract}
Resumo
O pneumoperitônio consiste na insuflação da cavidade abdominal com gás para realização de técnicas cirúrgicas laparoscópicas. Todavia, apesar de suas indicações, o estabelecimento do pneumoperitônio está associado a diversas alterações hemodinâmicas e respiratórias. O presente estudo objetivou investigar a ocorrência de alterações eletrocardiográficas decorrentes da insuflação peritoneal com $\mathrm{CO}_{2}$ durante a videolaparoscopia em gatas. Para isto, foram utilizadas 10 gatas adultas e hígidas, premedicadas com cloridrato de cetamina $\left(15 \mathrm{mg} \cdot \mathrm{kg}^{-1}\right)$ e midazolam $\left(0,2 \mathrm{mg} \cdot \mathrm{kg}^{-1}\right)$ e anestesiadas com isoflurano, submetidas a pneumoperitônio durante ovariossalpingohisterectomia (OSH) eletiva por videolaparoscopia. O traçado eletrocardiográfico foi avaliado em diferentes momentos através de eletrocardiógrafo Funbec ${ }^{\circledR}$ ECG5. Os dados obtidos foram analisados por análise de variância (ANOVA) e posterormente analisados pelo teste $t$ de Student e teste de Tukey, considerando significativos valores de $p<0,05$. As variações observadas no eletrocardiograma não representaram diferenças estatísticas quando analisadas pelos testes estatísticos realizados. Conclui-se que o pneumoperitônio com $\mathrm{CO}_{2}$ a uma pressão de $15 \mathrm{mmHg}$ não gera alterações eletrocardiográficas em gatas anestesiadas com isoflurano. Novos estudos abrangendo também a avaliação de eletrólitos e do pH sanguíneo podem ser realizados para melhor entendimento dos mecanismos envolvidos na insuflação peritoneal com $\mathrm{CO}_{2}$ durante a videocirurgia.

Palavras-Chave: Laparoscopia; Equilíbrio ácido-básico, Eletrólitos.
\end{abstract}

Como citar esse artigo. Silva, JDRT, Antunes F. Investigação de alterações eletrocardiográficasemgatas(Feliscatus) submetidas a pneumoperitônio. Revista Saúde. de 2016 Jul/Dez.; 07 (2): 16-21.

\begin{abstract}
Pneumoperitoneum constitutes insufflation of the abdominal cavity with gas to perform surgical laparoscopic techiques. However, despite its indications, the establishment of pneumoperitoneum is associated with several haemodynamic and respiratory changes. This study investigated occurrence of electrocardiographic changes due to peritoneal insufflation with $\mathrm{CO}_{2}$ during videolaparoscopy in cats brought to the veterinary school for elective surgery. Ten adult healthy female cats were premedicated with ketamine hydrochloride $(15 \mathrm{mg} \cdot \mathrm{kg}-1)$ and midazolam $(0,2 \mathrm{mg} \cdot \mathrm{kg}-1)$ and anesthetized with isoflurane and submitted to pneumoperitoneum during elective oophorus-salpingo-hysterectomy by videolaparoscopy. The electrocardiographic layout was assessed in different moments by a Funbec ${ }^{\circledR}$ ECG5 electrocardiogram machine. Data obtained were analysed by ANOVA and post test by Student's t-test and Tukey's test, considering as significant $\mathrm{p}<0,05$ values. The changes observed in electrocardiogram did not represent statistical differences when analysed by statistical tests performed on our methodology. In conclusion pneumoperitoneum with $\mathrm{CO}_{2}$ at $15 \mathrm{mmHg}$ does not cause eletrocardiographic changes in cats anesthetized with isoflurane. New researches including electrolytes and blood $\mathrm{pH}$ assessment could be performed to better allow understanding of the acid base mechanisms involved on peritoneal insufflation with $\mathrm{CO}_{2}$ during the videosurgery.

Keywords: Laparoscopy; Acid-base balance; Electrolytes.
\end{abstract}

\section{Introdução}

Um dos requisitos básicos para realização da laparoscopia inclui a insuflação peritoneal com gás e criação do pneumoperitônio, que equivale em vídeocirurgia ao campo cirúrgico da cirurgia convencional. O preenchimento da cavidade abdominal com gás é fundamental para viabilizar o trabalho do cirurgião na laparoscopia, já que a distensão abdominal e consequente separação dos órgãos daquela região promove o aumento do espaço de trabalho e aprimora a visualização das estruturas anatômicas ${ }^{1}$. Gases como o oxigênio, nitrogênio, óxido nitroso, argônio, hélio e até mesmo o ar ambiente já foram utilizados para realização do pneumoperitônio. Todavia, tem-se a preferência pelo dióxido de carbono $\left(\mathrm{CO}_{2}\right)$ por questões farmacocinéticas, facilidade de aquisição e segurança, uma vez que este não é um composto inflamável ${ }^{2}$. Segundo Oliveira ${ }^{3}$, quando $\mathrm{O}_{2}$ é introduzido na cavidade abdominal, não se mantém estático, difundindo-se através de

\footnotetext{
Afiliação dos autores:

† Médica Veterinária Autônoma.

† Residente de Anestesiologia Veterinária da Universidade Estadual do Norte Fluminense, Campos dos Goytacazes, RJ, Brasil.

$\S$ Mestranda - Programa de Pós-Graduação em Ciência Animal - Universidade Estadual do Norte Fluminense, Campos dos Goytacazes, RJ, Brasil

\| Mestre em Ciência Animal - Universidade Estadual do Norte Fluminense, Campos dos Goytacazes, RJ, Brasil.

O Professor Associado de Anestesiologia Veterinária da Universidade Estadual do Norte Fluminense, Campos dos Goytacazes, RJ, Brasil.
} 
todos os tecidos, entrando em equilíbrio com todos os compartimentos corporais. No entanto, alterações adversas foram observadas em vários órgãos e têm sido associadas com o aumento da carga sistêmica de $\mathrm{CO}_{2}$ e a elevação da pressão intra-abdominal que ocorre durante pneumoperitônio ${ }^{4}$.

A absorção do $\mathrm{CO}_{2}$ insuflado no peritônio para a circulação sistêmica durante o procedimento e a redução da complacência e capacidade funcional residual pulmonar colaboram para aumento na $\mathrm{PaCO}_{2}$. Se os parâmetros ventilatórios não estiverem ajustados, hipercapnia e acidose respiratória secundária irão se estabelecer ${ }^{5}$. Segundo Mergh e Oliveira ${ }^{6}$, ocorrem efeitos deletérios nas funções cardiovascular, pulmonar, renal e metabólica. A distensão abdominal causa deslocamento cranial do diafragma, resultando em redução dos volumes pulmonares, incluindo a capacidade residual funcional. A complacência pulmonar também se reduz e a resistência das vias aéreas aumenta, levando a uma maior pressão de vias aéreas (PVA) para qualquer volume corrente (VC) fornecido, com aumento do risco de alterações hemodinâmicas e de barotrauma durante ventilação controlada com pressão positiva intermitente (PPI). Estas alterações resultam em hipóxia e/ou hipercarbia. ${ }^{7,8,9}$

A perfusão tecidual inadequada leva à acidose metabólica, que pode ser detectada através de gasometria ou através de elevação dos níveis séricos de lactato. Esta situação é potencialmente deletéria, pois promove diminuição da contratilidade cardíaca, do índice cardíaco e da responsividade vascular periférica, além de proporcionar aparecimento de arritmias ventriculares ou dificultar o controle de arritmias já existentes. ${ }^{10}$ Além disso, a acidose metabólica leva à hipercalemia, devido à alta concentração extracelular de $\mathrm{H}^{+}$que resulta no movimento deste íon para dentro da célula e do potássio para fora da célula para que a eletroneutralidade seja mantida. ${ }^{11}$ Condições de hipercalemia predispõem a alterações como intervalos Q-T e segmento ST supranivelados no eletrocardiograma e arritmias. ${ }^{12}$

Diantedasalteraçõeshemodinâmicaseeletrolíticas supracitadas promovidas pelo pneumoperitônio e a possível repercussão das mesmas sobre o traçado eletrocardiográfico, o presente estudo teve como objetivo investigar as alterações eletrocardiográficas decorrentes do pneumoperitônio com $\mathrm{CO}_{2}$ em gatas submetidas a videolaparoscopia.

\section{Material e métodos}

Pararealizaçãodo experimento foram selecionadas 10 gatas adultas, pesando entre 2,8 e $4,1 \mathrm{~kg}$,destinadas ao setor de cirurgia do Hospital Veterinário da Universidade Estadual do Norte Fluminense Darcy Ribeiro (UENF) para ovariossalpingohisterectomia (OSH) eletiva. Os animais foram previamente avaliados através de exames clínicos e laboratoriais (eritrograma, leucograma, alanina transaminase, aspartato transaminase, fosfatase alcalina, ureia e creatinina) de modo a garantir a seleção de animais em condição de higidez.

Todos os animais foram submetidos a um protocolo padrão quanto à abordagem anestésica e cirúrgica. A medicação pré-anestésica foi realizada com $15 \mathrm{mg} . \mathrm{kg}^{-1}$ de cloridrato de cetaminaem associação com 0,2 mg. $\mathrm{kg}^{-1}$ de midazolam por via intramuscular. A indução anestésica foi realizada com isoflurano por máscara. Fluidoterapia de manutenção com ringer com lactato foi instituída a uma taxa de 10 ml.kg.hora ${ }^{-1}$ por via intravenosa no período transcirúrgico.

A videolaparoscopia foi realizada com os animais em posição de supino (decúbito dorsal), com insuflação intraperitoneal a uma pressão de 15 $\mathrm{mmHg}$. Os parâmetros vitais foram monitorados com monitor multiparamétricoAdvisor ${ }^{\circledR}$ e o traçado eletrocardiográfico foi avaliado com aparelho de eletrocardiograma Funbec $^{\circledR}$ ECG5. As avaliações foram realizadas nos seguintes momentos: M0 (antes do pneumoperitônio), M1 (imediatamente após a instituição do pneumoperitônio) e M2, M3 e M4 (em intervalos de 10 minutos após o estabelecimento do pneumoperitônio).

Ao término das avaliações, os dados foram formatados e submetidos à análise de variância (ANOVA) e posteriormente analisados pelo teste $t$ de Student e teste de comparação de médias (Tukey), considerando $p<0,05$. Todos os cálculos foram realizados com os softwares Sigma-Stat e Sigma-Plot versão 9.0.

\section{Resultados e Discussão}

O estudo eletrocardiográfico não evidenciou alteração em onda $\mathrm{P}$ dos animais estudados, a qual manteve-se em níveis regulares durantes todos os momentos avaliados, conforme observa-se no gráfico 1. Os resultados apresentados nos gráficos 2,3 e 4 não apontaram diferenças estatísticas nas variações de amplitude do complexo QRS e intervalos P-R e Q-T monitorados no eletrocardiograma em nossos experimentos. Estes achados corroboram com os resultados de O'Learyet al. ${ }^{13}$, que demonstraram que a insuflação peritoneal com $\mathrm{CO}_{2}$ a uma pressão de 14 $\mathrm{mmHg}$ em humanos induz aumento da pressão arterial média, mas nenhuma alteração no segmento ST foi encontrada em pacientes hígidos, não obesos e sem de doença cardíaca em colecistectomias laparoscópicas eletivas. Em uma série de 16 pacientes, apenas um deles apresentou alterações no intervalo ST do ECG durante a exsuflação do $\mathrm{CO}_{2}$ intraperitoneal. Todavia tais alterações não provocaram sequelas pós-operatórias. 
Gráfico 1. Avaliação da amplitude da onda $\mathrm{P}$, em $\mathrm{mV}$, durante o procedimento.

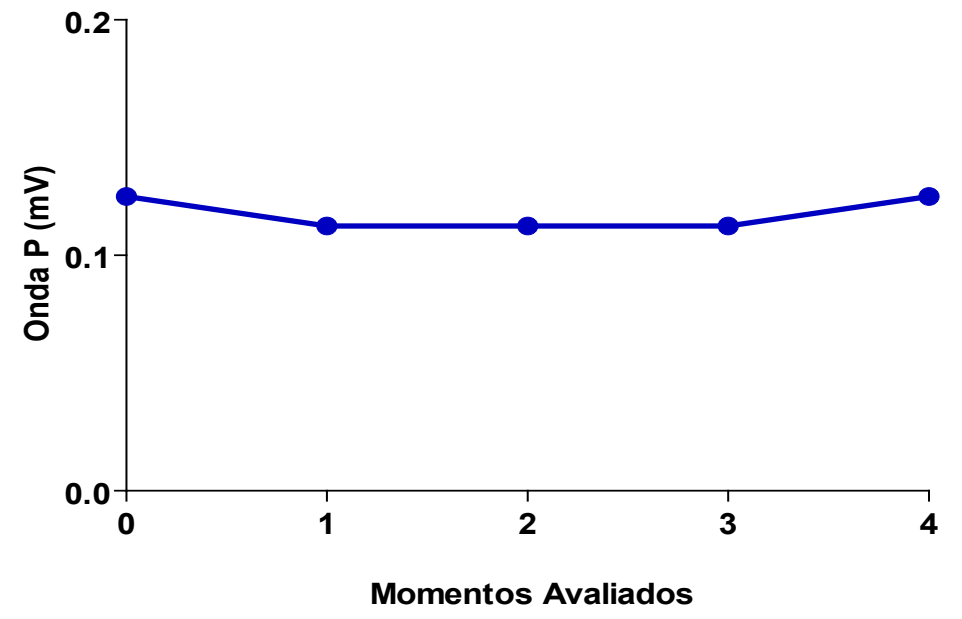

Gráfico 2. Amplitude das ondas do complexo QRS em mV no eletrocardiograma.

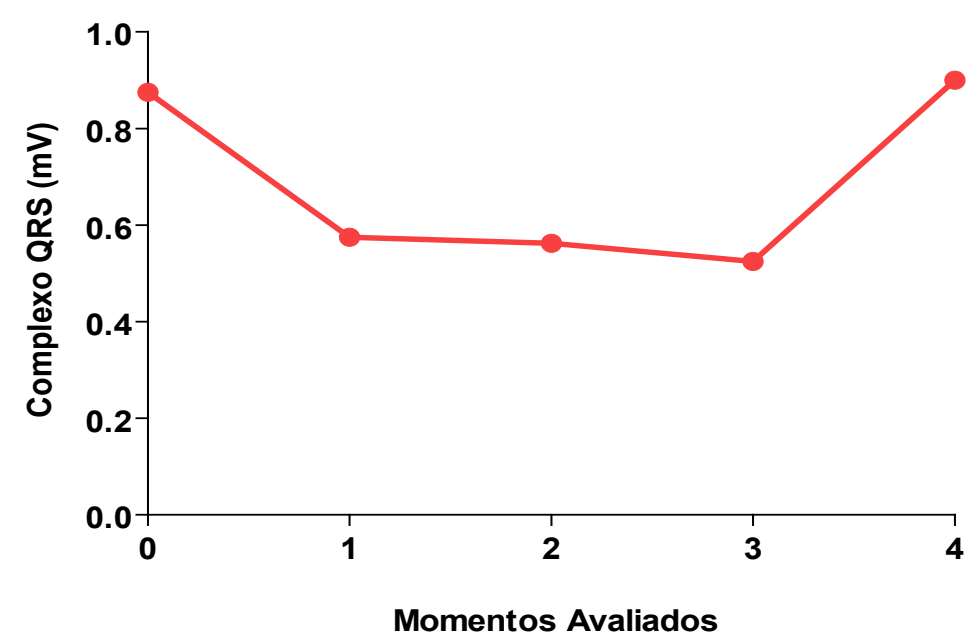

Gráfico 3. Intervalo entre as ondas P e R, em segundos, nos momentos avaliados

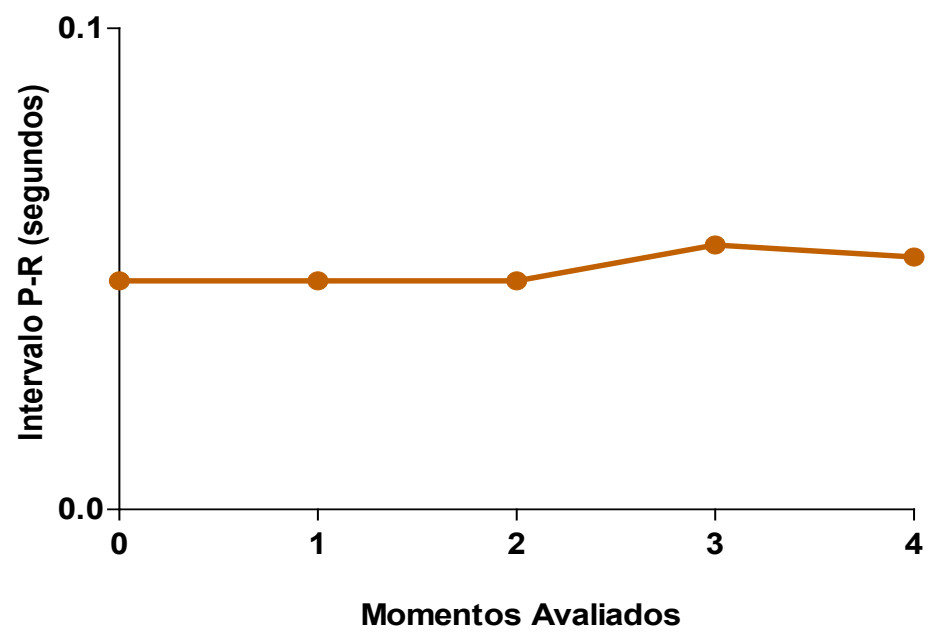


Gráfico 4. Avaliação do intervalo Q-T, em segundos, no eletrocardiograma.

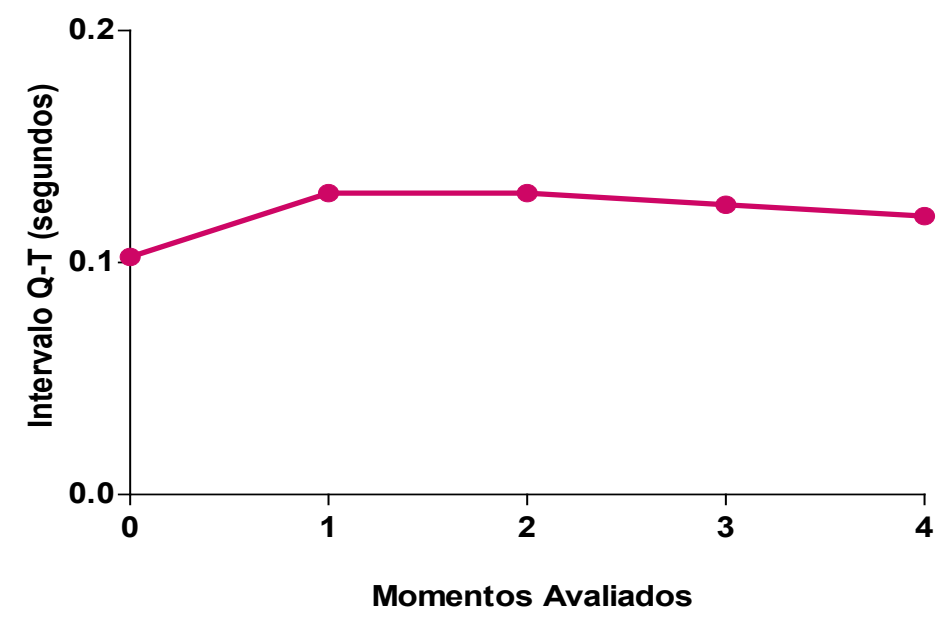

Gráfico 5. Amplitude da onda T, em mV, nos momentos avaliados.

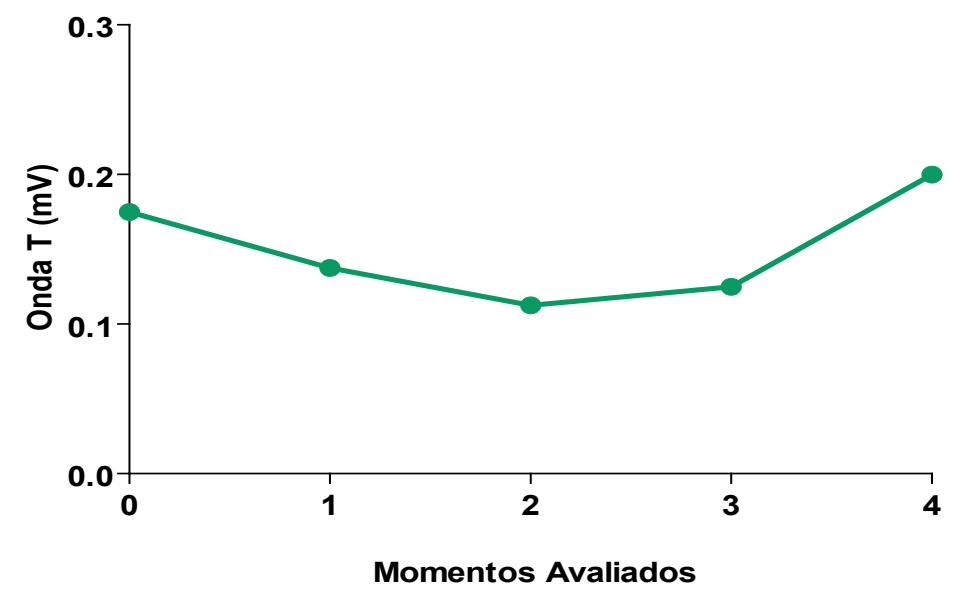

Observa-se também que, conforme o gráfico 5, não houve influência do pneumoperitônio sobre a onda $T$ dos animais avaliados em nossos estudos.

Nos estudos de Perneret al. ${ }^{14}$, as concentrações plasmáticas de potássio não foram afetadas pelo pneumoperitônio com $\mathrm{CO}_{2}$ em pacientes jovens e relativamente sadios durante procedimentos laparoscópicos curtos. Os mesmos autores relatam que o pneumoperitônio com $\mathrm{CO}_{2}$ por tempo prolongado em pacientes mais velhos induziu apenas um pequeno aumento nas concentrações de potássio no plasma, que não representou significância estatística e clínica, apesar da ocorrência de acidose metabólica progressiva. Nossos resultados condizem com estes achados, uma vez que os animais utilizados em nossos experimentos haviam sido previamente examinados e avaliados para a seleção daqueles que apresentassem em condições de higidez para realização do procedimento cirúrgico e estes não apresentaram alterações clínicas condizentes com quadro de acidose.

O desenvolvimento de acidose metabólica progressiva durante o pneumoperitônio com $\mathrm{CO}_{2}$ observado em colecistectomias realizadas por Peneret al. ${ }^{14}$ éexplicado pelos autores pela redução da perfusão periférica, uma vez que, em seus achados, houve correlação inversa entre $\mathrm{o} \mathrm{pH}$ e a pressão arterial média, sendo este segundo, um reflexo das alterações na resistência vascular periférica ocorridas durante a insuflação peritoneal com $\mathrm{CO}_{2}$. Demiroluk et al. ${ }^{5}$, encontraram resultados semelhantes aos supracitados. Houve aumento estatisticamente significante nos níveis de potássio associado a hipercapnia no período após o pneumoperitônio. Porém, o aumento de potássio descrito não foi clinicamente relevante e não gerou nenhuma alteração eletrocardiográfica em nenhum dos pacientes estudados. 
Como observa-se no gráfico 6 , houve diferença estatística na comparação das médias obtidas em M2 (157,5 bpm) e M3 (152,5 bpm) com os valores mensurados inicialmente em M0 (225 bpm) em nossos ensaios. Podemos afirmar, portanto, que houve redução da frequência cardíaca nos animais estudados. Todavia, o número de batimentos por minuto se manteve dentro dos parâmetros normais para a espécie em questão ${ }^{15}, o$ que pode ter colaborado para a manutenção de um débito cardíaco adequado e, consequentemente, compensação de quaisquer alterações na pressão arterial média e na resistência vascular periférica que viessem a causar

Gráfico 6. Avaliação da frequência cardíaca dos animais submetidos ao pneumoperitônio.

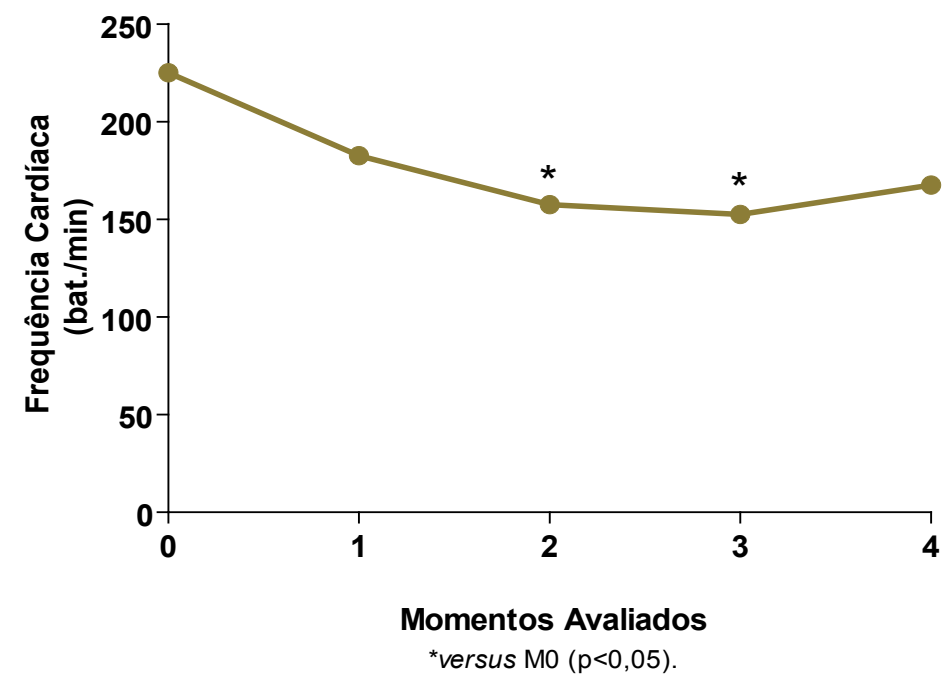

hipoxemia e eventuais alterações no $\mathrm{pH}$.

Segundo Hanly et al. ${ }^{16}$, a insuflação abdominal com $\mathrm{CO}_{2}$ causa acidose peritoneal local sem afetar o estado ácido-básico sistêmico em animais adequadamente ventilados. Em contrapartida, Volz et al. ${ }^{17}$ demonstraram que alterações sistêmicas como a acidose, aumento da $\mathrm{PCO}_{2}$ e a produção de $\mathrm{CO}_{2}$ são atribuídas à absorção e efeitos locais do $\mathrm{CO}_{2}$ na região peritoneal. Entretanto, este efeito somente ocorreu quando houve associação do $\mathrm{CO}_{2}$ com a alta pressão intraperitoneal, não sendo, portanto, atribuível a distúrbios de microcirculação no peritônio ou alterações pulmonares consequentes do aumento na pressão intraabdominal, pois a alta pressão intra-abdominal isolada não foi capaz de produzir os mesmos efeitos. Segundo o mesmo autor, quase todas as alterações desfavoráveis no equilíbrio ácido-básico podem ser evitadas com a redução da pressão de manutenção do $\mathrm{CO}_{2}$ insuflado na cavidade peritoneal. Diante destas informações, é importante salientar que, a ocorrência ou não de alterações eletrocardiográficas depende de uma série de fatores que, se corrigidos por parte da equipe cirúrgica ou compensados fisiologicamente pelo paciente, não resultarão em alterações clínicas como acidose e arritmias, por exemplo.

\section{Conclusão}

As alterações observadas no traçado

eletrocardiográfico não representaram diferenças estatísticas quando analisadas pelos testes previstos em nossa metodologia. Todavia, correlacionando nossos achadoscomaliteraturaexistenteatéopresentemomento, concluímos que em gatas hígidas e sem histórico de doença cardíaca pré-existente, anestesiadas e em posição de supino, a realização do pneumoperitônio com $\mathrm{CO}_{2}$ a uma pressão de $15 \mathrm{mmHg}$, não produz alterações eletrocardiográficas relevantes. Ainda assim, novos estudos avaliando, além do eletrocardiograma, também o pH sanguíneo e as dosagens sanguíneas de eletrólitos como potássio e cálcio, além de gases sanguíneos, são necessários para o melhor entendimento da influência do aumento da pressão intra-abdominal causado pelo pneumoperitôniocom $\mathrm{CO}_{2}$ sobre o eletrocardiograma de gatas submetidas a cirurgia laparoscópica.

\section{Referências}

1. Nesek-Adam V, Rasic Z, Kos J, Vnuk D. Aminotransferases after experimental pneumoperitoneum in dogs. Acta Anaesthesiol Scand, 2004; 48(7):862-866

2. Koivusalo AM, Lindgren L. Effects of carbon dioxide pneumoperitoneum for laparoscopic cholecystectomy. Acta Anaesthesiol Scand, 2000; 44: 834841 .

3. Oliveira CRD. Anestesia para cirurgia videolaparoscópica. RevBrasVideocir, 2005; 3(1): 32-42.

4. Yilmaz S,Polat C,Kahraman A,Koken T,Arikan Y,Dilek ON,Gökçe O. The comparison of the oxidative stress effects of different gases and intraabdominal pressures in an experimental rat model. J Laparoendosc Adv Surg 
Tech A, 2004;14(3):165-168.

5. Demiroluk S, Salihoglu Z, Bozkurt P, Hayirlioglu M, Kise Y. Effect of pneumoperitoneum on the level of plasma potassium. M E J Anesth, 2007; 19(1): $61-70$.

6. Mergh ACM, Oliveira CHS; Alterações pulmonares na cirurgia videolaparoscópica. Rev Bras Anestesiol, 1996; 46(4): 289-294.

7. Hanly EJ, Fuentes JM, Aurora RA, Bachman SL, Maio A, Marohn MR et al. Carbon dioxide pneumoperitoneum prevents mortality from sepsis. Surg Endosc, 2006; 20(9): 1482-1487.

8. Meininger D, Westphal K, Bremerich DH, Runkel H, Probst M, Zwissler B et al.Effects of Posture and Prolonged Pneumoperitoneum on Hemodynamic Parameters during Laparoscopy. World J Surg, 2008; 32(7):.1400-1405.

9. Naude GP, Bongard FS. Helium insufflation in laparoscopic surgery. Endosc Surg Allied Technol, 1995; 3(4): 183-186.

10. Knobel E. Choque cardiogênico. Arq Bras Cardiol, 1999; 72(4): 405413.

11. Schaefer TJ, Wolford RW. Disorders of potassium. Emerg Med Clin North Am, 2005; 23(3): 723-747.

12. Évora PRB, Reis CL, Ferez MA, Conte DA, Garcia LV. Distúrbios do equilíbrio hidroeletrolítico e do equilíbrio ácido-básico - Uma revisão prática. Medicina (Ribeirão Preto), 1999; 32: 451-469.

13. O'Leary E, Hubbard K, Tormey W, Cunningham AJ. Laparoscopic cholecystectomy:haemodynamic and noroendocrine responses after pneumoperitoneum and changes in position. Br J Anaesth, 1996; 76: 640644.

14. Perner A, Bugge K, Lyng KM, Schulze S, Kristensen A, Bendtsen A; Changes in plasma potassium during carbon dioxide pneumoperitoneum. $\mathrm{Br}$ J Anaesth, 1999; 82(1): 137-139.

15. Camacho AA, Mucha CJ. Semiologia do sistema circulatório de cães e gatos. In: Feitosa, FLF. Semiologia Veterinária: A arte do diagnóstico. 2 ed. São Paulo: Roca, 2008. 842 p.

16. Hanly EJ, Fuentes JM, Aurora AR, Shih S, Marohn MR, Maio A, Talamini MA. Abdominal insufflation with $\mathrm{CO} 2$ causes peritoneal acidosis independent of systemic pH. J Gastrointest Surg, 2005; 9: 1245-1252.

17. Volz J, Köster S, Weis M, Schimidt R, Urbaschek R, Melchert F, Albercht M. Pathophysiologic features of a pneumoperitoneum at laparoscopy: A swine model. Obstet Gynecol, 1996; 174: 132-140. 